\title{
Macrophages Are Alternatively Activated in Patients with Endometriosis and Required for Growth and Vascularization of Lesions in a Mouse Model of Disease
}

\author{
Monica Bacci, ${ }^{*}$ Annalisa Capobianco, ${ }^{*}$ \\ Antonella Monno, ${ }^{*}$ Lucia Cottone, ${ }^{*}$ \\ Francesca Di Puppo, ${ }^{\dagger}$ Barbara Camisa, ${ }^{\ddagger}$ \\ Margherita Mariani, ${ }^{\ddagger}$ Chiara Brignole, ${ }^{\S}$ \\ Mirco Ponzoni, ${ }^{\S}$ Stefano Ferrari, ${ }^{\dagger}$ \\ Paola Panina-Bordignon, ${ }^{\ddagger}$ Angelo A. Manfredi, ${ }^{*}$ \\ and Patrizia Rovere-Querini*

\begin{abstract}
From the Clinical Immunology and Innate Immunity and Tissue Remodelling Units, ${ }^{*}$ Division of Regenerative Medicine, Stem Cells, and Gene Therapy, and the Department of Gynaecology and Obstetrics, ${ }^{\dagger}$ all from the H. San Raffaele Scientific Institute and Vita Salute San Raffaele University, Milano; Bioxell Spa, Milano; and the Laboratory of Oncology, ${ }^{\Im}$ G. Gaslini Children's Hospital, Genova, Italy
\end{abstract}

The mechanisms that sustain endometrial tissues at ectopic sites in patients with endometriosis are poorly understood. Various leukocytes, including macrophages, infiltrate endometriotic lesions. In this study, we depleted mouse macrophages by means of either clodronate liposomes or monoclonal antibodies before the injection of syngeneic endometrial tissue. In the absence of macrophages, tissue fragments adhered and implanted into the peritoneal wall, but endometriotic lesions failed to organize and develop. When we depleted macrophages after the establishment of endometriotic lesions, blood vessels failed to reach the inner layers of the lesions, which stopped growing. Macrophages from patients with endometriosis and experimental mice, but not nonendometriotic patients who underwent surgery for uterine leiomyomas or control mice, expressed markers of alternative activation. These markers included high levels of scavenger receptors, CD163 and CD206, which are involved in both the scavenging of hemoglobin with iron transfer into macrophages and the silent clearance of inflammatory molecules. Macrophages in both inflammatory liquid and ectopic lesions were equally polarized, suggesting a critical role of environmental cues in the peritoneal cavity.
Adoptively transferred, alternatively activated macrophages dramatically enhanced endometriotic lesion growth in mice. Inflammatory macrophages effectively protected mice from endometriosis. Therefore, endogenous macrophages involved in tissue remodeling appear as players in the natural history of endometriosis, required for effective vascularization and ectopic lesion growth. (Am J Pathol 2009, 175:547-556; DOI: 10.2353/ajpath.2009.081011)

Endometriosis is a common disease, characterized by the persistence and growth of vascularized endometrial tissue at ectopic sites, typically the pelvis, and associated with pelvic pain and infertility. ${ }^{1-3}$ Endometriotic lesions possibly originate from shed endometrial tissue that reaches the abdominal cavity via the Fallopian tubes during menstruation. ${ }^{4}$ This process, referred to as retrograde menstruation, occurs in most women during the fertile years. In women who develop endometriosis, endometrial tissue attaches to the peritoneal wall. The following steps include invasion of the underlying basement membrane and recruitment of novel vessels from the peritoneal vasculature. ${ }^{5,6}$

The events underlying the susceptibility of menstruating women to endometriosis are obscure. The ability of shed endometrium, to attach and to infiltrate the peritoneum, to survive, and to recruit vessels are possibly limiting steps. Neo-angiogenesis physiologically takes place during wound healing as a consequence of macrophage activation. ${ }^{7,8}$ Macrophages represent a major source of neo-angiogenic signals in solid tumors, which depend on newly formed vessels for their growth and spreading. In solid tumors, macrophages also deliver

Supported by the AIRC, by the MIUR, and by the Ministero della Salute. Accepted for publication April 28, 2009.

Address reprint requests to Patrizia Rovere-Querini, Istituto Scientifico Ospedale San Raffaele, DIBIT, 3A1, via Olgettina 58, 20132, Milano Italy. E-mail: rovere.patrizia@hsr.it. 
signals that limit apoptosis under hypoxic conditions and facilitate local infiltration and metastatic spreading. ${ }^{9}$

Recombinant cytokines or microbial products elicit the in vitro differentiation of macrophage precursors toward such a reparative phenotype (also called alternative or M2 activation) or toward inflammatory M1 cells, better suited to fight invading pathogens. The signals that commit precursors in living tissues to polarize toward M1 or M2 macrophages, in conditions of infection, sterile injury, or neoplastic transformation, are only beginning to be elucidated. Interestingly, a heterogeneity in response to innate signals among the population has been demonstrated, suggesting that environmental signals result in diverse patterns of macrophage activation in different subjects. ${ }^{10,11}$

Differences in macrophage activation in the peritoneal cavity could therefore be involved in the susceptibility/ resistance to endometriosis: a "permissive" macrophage activation would facilitate the survival of shed endometrial tissue via the production of trophic and anti-apoptotic signals, facilitate invasion of the mesothelial layer via interference with the balance between metalloproteinase and their inhibitors, ${ }^{12}$ and could be later on required for neo-angiogenesis. In the current study, using an experimental mouse model, we verified the hypothesis that macrophage activation is involved in the establishment, survival, and spreading of ectopic lesions from shed endometrium. We finely characterized macrophages in the peritoneal fluid and in established endometriotic lesions, in experimental mice and human, and found that they share evident features of alternative, disease-permissive, activation.

\section{Materials and Methods}

\section{Mice}

All procedures were performed in the animal facility of $\mathrm{H}$ San Raffaele Scientific Institute (Italy) in accordance with European Union guidelines and with the approval of the Institutional Animal Care and Use Committee of our Institution. Eight-week-old female Balb/C mice were purchased from Charles River (Calco, Italy). We used 10 animals per experimental group for each independent experiment.

\section{Model of Endometriosis}

Mice were initially treated subcutaneously with estradiol benzoate (3 $\mu \mathrm{g} / \mathrm{mouse}$, Intervet s.r.l., Milan, Italy). Seven days later mice were sacrificed (one donor mouse for every two to be challenged with endometrium), uteri were removed, seeded in a Petri dish containing warmed saline, and split longitudinally with a pair of scissors. Each uterine horn was identically processed, including isolation of endometrial tissue and its careful mechanical disruption in small cell aggregate suspension, of a maximal diameter that was consistently lower than $1 \mathrm{~mm}$, before intraperitoneal injection of an experimental/control mice pair. ${ }^{13,14}$ By this approach each mouse received the suspension derived from a half uterus. Twelve days after endometrial tissue injection, mice were euthanized individually by cervical dislocation. The abdominal cavity was immediately opened and lesions excised and processed for disease assessment or immunohistochemistry evaluation. The extent of endometriosis was evaluated by assessing the dry weight of all lesions from each mice, as described. ${ }^{13,14}$

\section{In Vivo Macrophage Depletion}

To verify the role of macrophages in the establishment and growth of ectopic endometrial lesions, mice were treated i.p. with the depleting anti-F4/80 Ab $(0.80 \mu \mathrm{g} / \mathrm{g} /$ mice clone Cl: A3-1, SeroTec, Oxford, UK) or PBS, or with liposomes containing either clodronate (Sigma Aldrich, St Louis, MO) or PBS, prepared as described..$^{15}$ When indicated, depleting agents were injected intraperitoneally. The anti-F $4 / 80$ depleting $m A b$ was injected at day -2 , the treatment was repeated at day $0,+2,+4,+6,+8,+10$ after uterus transfer. Liposomes containing PBS or clodronate were injected at days $-1,0,+4$, and +8 after endometrial tissue transfer. The final clodronate liposome suspension contained $5 \mathrm{mg}$ of clodronate $/ \mathrm{ml}$. Both treatments kill monocytes and macrophages, while are not per se otherwise toxic. Control mice were injected i.p. with PBS. To assess the effect of macrophage depletion on ectopic endometrial tissue early survival and adhesion to the peritoneal cavity, depletion was performed only at day 0 . To assess the effect of macrophage depletion on already established lesions, the treatment was performed exclusively at days $+4,+8$, ie, after lesion engraftment. To verify the efficacy of the treatment, blood or peritoneal fluid was retrieved at various times and analyzed by flow cytometry. Samples (30 $\mu$ l) were incubated for $10 \mathrm{~min}$ utes at room temperature with PBS containing 10\% fetal calf serum. Phycoerythrin-conjugated anti-F4/80 mAb (clone Cl: A3-1, SeroTec) and/or allophycocyanin-conjugated anti-CD11b mAb (clone M1/70, BD Biosciences) (2 $\mu \mathrm{l} / \mathrm{sample}$ ) were added for 20 minutes. Red blood cell lysis buffer $\left(0.15 \mathrm{M} / \mathrm{L} \mathrm{NH} \mathrm{N}_{4} \mathrm{Cl}, 1 \mathrm{mmol} / \mathrm{L} \mathrm{KHCO}_{3}\right.$, and 0.1 $\mathrm{mmol} / \mathrm{L} \mathrm{Na}_{2} \mathrm{EDTA}$ ) was added for 10 minutes at room temperature before analysis (FACS Calibur flow cytometer and CellQuest software, BD Biosciences).

\section{Propagation of Polarized Macrophages From Mouse Bone Marrow Precursors}

Bone marrow precursors from Balb/C female mice were retrieved and cultured for 7 days in $\alpha$-MEM (GIBCO, Invitrogen, Carlsbad, CA) containing 10\% fetal bovine serum (Lonza, Basel, Switzerland) and recombinant murine $(\mathrm{rm})$ macrophage-colony-stimulating factor $(100 \mathrm{ng} /$ $\mathrm{ml}$ ), yielding non polarized macrophages (MO). Cells were then further cultured for 48 hours in the presence of rm interferon- $\gamma$ ( $50 \mathrm{ng} / \mathrm{ml}$ ) (PeproTech, Rocky Hill, NJ) to obtain inflammatory/M1 macrophages or with rm macrophage-colony-stimulating factor $(10 \mathrm{ng} / \mathrm{ml})+\mathrm{rm}$ interleukin (IL)-10 (10 ng/ml) for 96 hours to obtain alternatively activated $\mathrm{M} 2$ cells. Polarization was verified by flow 
cytometry as described. ${ }^{16}$ The secretion of prototypical M1 and M2 cytokines, such as tumor necrosis factor (TNF)- $\alpha$ and IL-10 respectively, was verified by enzymelinked immunosorbent assay (R\&D Systems).

\section{In Vivo Delivery of Polarized Macrophages}

In selected experiments undifferentiated (M0), M1, and M2 macrophages generated in vitro as above, were injected in the peritoneal cavity of recipients mice at days $-2,0$, and +2 . At day 0 , mice received the intraperitoneal injection of endometrial tissue and were sacrificed at day +12 . Lesions were excised and processed for disease assessment or immunohistochemistry evaluation as above.

\section{Macrophages Assessment in the Mouse Peritoneal Liquid}

Peritoneal cells were retrieved by peritoneal lavage of treated and untreated animals, and resuspended in cold PBS containing $2 \mathrm{mmol} / \mathrm{L}$ EDTA. Cell viability, verified by trypan blue exclusion, was typically $>98 \%$. Cells were stained with allophycocyanin-conjugated anti-CD11b Ab (BD, Pharmingen), phycoerythrin-conjugated anti-F4-80 $\mathrm{Ab}(\mathrm{R} \& \mathrm{D})$, or with purified unlabeled rat anti-mouse antiCD206 Ab and purified antibody rabbit anti-mouse antiCD163 (Serotec). Phycoerythrin-conjugated goat anti-rat or fluorescein isothiocyanate (FITC)-conjugated goat anti-rabbit $\mathrm{Ab}$ were used as second step reagents before analysis by 2- and 4-parametric flow cytometry.

\section{Immunohistochemistry of Murine Lesions}

Endometriotic lesions were frozen in liquid $\mathrm{N}_{2}$-cooled isopentane. Serial 6-mm thick sections were fixed with $4 \%$ paraformaldehyde (10 minutes at room temperature) and successively treated with $0.3 \% \mathrm{H}_{2} \mathrm{O}_{2}$ (10 minutes at room temperature) to quench endogenous peroxidase activity. To evaluate macrophage infiltration, tissue sections were incubated in PBS $+5 \%$ bovine serum albumin for 1 hour at room temperature and then overnight at $4^{\circ} \mathrm{C}$ with rat antimouse CD68 mAb (2 mg/ml, clone FA-11, SeroTec), CD163 ( $1 \mathrm{mg} / \mathrm{ml}$, clone M-96, Santa Cruz Biotechnology, Inc, Santa Cruz, CA), and CD206 (1 mg/ml, clone MR5D3, SeroTec). Endothelial cells were identified by staining with anti-mouse CD31 mAb (Serotec). Primary Abs were revealed using biotin conjugated anti-rat polyclonal lgG $(1.5 \mathrm{mg} / \mathrm{ml}$, eBiosciences, San Diego, CA) and R.T.U horseradish peroxidase streptavidin (Vector Laboratories, Burlingame, CA), which was detected using Vector NovaRED substrate kit (Vector Laboratories). Slides were counterstained with hematoxylin and examined under a Nikon Eclipse 55i microscope (Nikon, Tokyo, Japan). Images were captured with Digital Sight DS-5 M digital camera (Nikon) using Lucia G software (Laboratory Imaging, Prague, CZ). Parallel slides in which primary Abs had been omitted were identically processed and used as negative controls.

\section{Patients and Controls}

Venous blood, peritoneal liquid, and tissue samples (lesions and disease-free peritoneum) were obtained between September 2006 and June 2007 from 23 patients with patho-

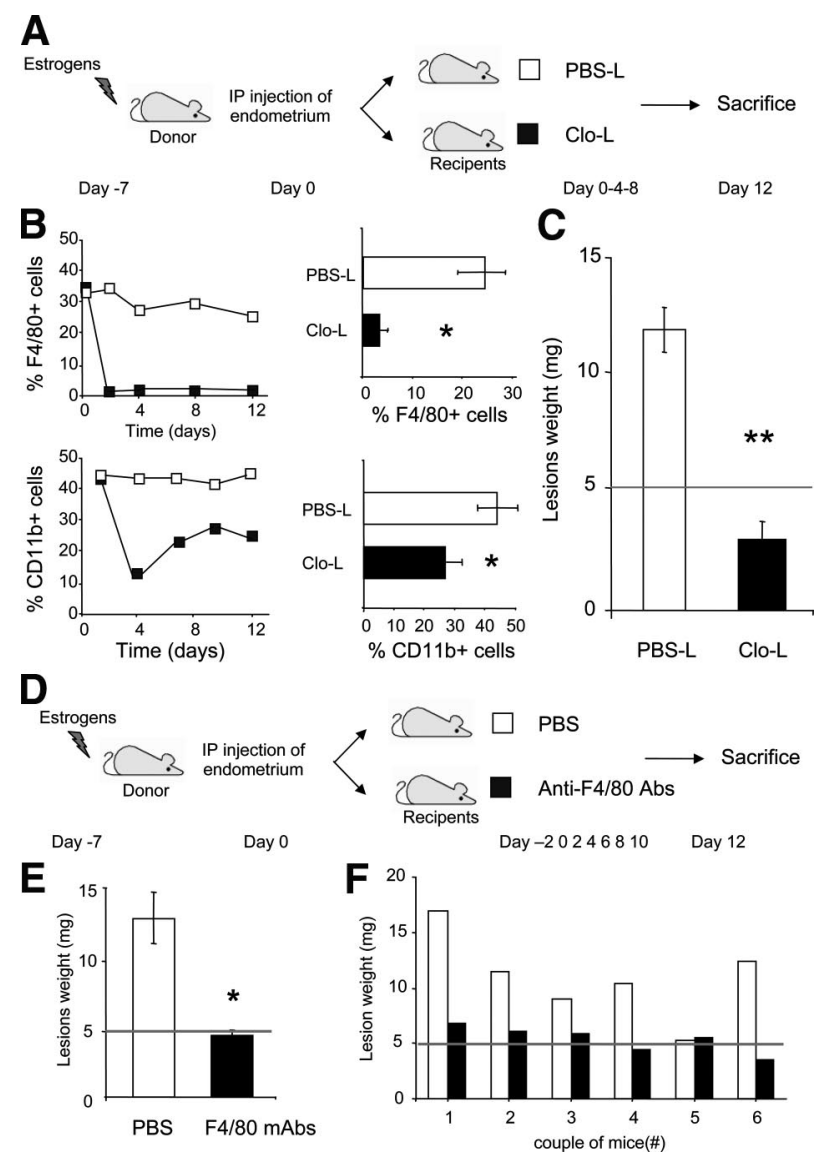

Figure 1. Peritoneal macrophages are required for endometriosis establishment. A: Estradiol benzoate-treated female mice were sacrificed and uteri removed and split. Endometrial tissue was isolated and mechanically disrupted before intraperitoneal injection of an experimental/control mice pair. Control mice (open squares) and depleted mice (filled squares) were treated i.p. at day 0,4 , and 8 with liposomes containing PBS (PBS-L) or clodronate (Clo-L) respectively. Lesions were evaluated 12 days after endometrial tissue injection. B: The percentage of peritoneal $\mathrm{F} 4 / 80^{+}$and of $\mathrm{CD} 11 \mathrm{~b}+$ macrophages was evaluated by flow cytometry at different time points (left panels). Right panels represent the quantitative expression of the results obtained at day 12, expressed as percentage of positive cells in mice treated with PBS-L (open columns) or Clo-L (filled columns) respectively. C: The dry weight of endometriotic lesions from mice treated with PBS-L or with Clo-L was assessed at day 12. Results are expressed as mean of total weights of lesions from six independent experimental/control mouse pair \pm SEM. D: Estradiol benzoate-treated female mice were sacrificed and uteri removed and split. Endometrial tissue was isolated and mechanically disrupted before intraperitoneal injection of experimental/control mice pairs. Mice were treated with PBS (open squares) or F $4 / 80 \mathrm{mAb}$ mice (filled squares) i.p. 2 days before injection of syngeneic endometrial tissue (day -2$)$ and every other day since then (days $0,2,4,6,8,10$, and 12). Lesions were evaluated at day 12 , when mice were sacrificed. E: The dry weight of endometriotic lesions from mice treated with PBS (open columns) or with F4/80 mAb (filled columns) was assessed at day 12. Results are expressed as mean of total weights of lesions from six independent experimental/control mouse pair \pm SEM. F: The dry weight of endometriotic lesions from mice treated with PBS or with F4/80 mAb was assessed at day 12. Results from each independent experimental/control mouse pair, in which endometrial tissue from a single donor was split and injected in a mouse previously injected with PBS (open columns) or with $\mathrm{F} 4 / 80 \mathrm{mAb}$ (filled columns). Significantly different from control, ${ }^{*} P<0.05$ and ${ }^{* * *} P<0.01$. 
logically proven endometriosis and 13 matched controls undergoing surgery for uterine leyiomyomas, all followed in the Department of Obstetrics \& Gynaecology of the H San Raffaele Scientific Institute (Italy). The San Raffaele Ethical Committee approved the study protocol and all subjects provided written informed consent. Patients and controls were menstruating women between 20 and 45 years that did not receive hormonal and/or anti-inflammatory medications for at least 3 months before sample collection. The stage of disease was established according to the revised American Fertility Society (AFSr) classification. ${ }^{17}$ In our analysis, we considered three stages, mild (AFSr stages I-II; $n=5$ ), moderate (AFSr stage III; $n=8$ ), and severe (AFSr stage IV; $n=8)$.

\section{Monocyte/Macrophage Assessment in Human Samples}

Peripheral blood mononuclear cells were obtained by centrifugation over a ficoll density gradient (Ge Health
Care, Piscataway $\mathrm{NJ}$ ) and inflammatory cells within the peritoneal fluid were obtained by centrifugation after lysis of red blood cells with ammonium chloride $(0.15$

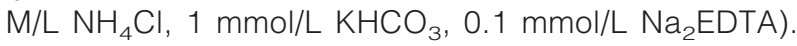
Viable cells, identified by trypan blue exclusion, were consistently $>90 \%$. 1 to $1.5 \times 10^{5}$ cells/sample were stained with: Percp-conjugated anti-CD45 and anti-CD14, phycoerythrin-conjugated anti-CD16, and anti-CD163, FITC-conjugated anti-CD206 anti-CD36, anti-CD86, anti-HLA-ABC, anti-HLA-DR mAbs, all from Becton Dickinson (BD Pharmingen, California). When indicated, cells were stained with purified anti-receptor for advanced glycation end products (rabbit polyclonal IgG, Santa Cruz Biotecnology) or with biotinylated anti$\alpha \vee \beta 3$ mAbs (Chemicon International). FITC-conjugated anti-rabbit Ab or FITC-conjugated streptavidin were used as second step reagents. Cells were analyzed by flow cytometry (FACS Calibur, Bekton Dickinson California). Analysis was performed using a FACS express version 3 software.

A

Estrogens

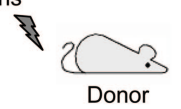
IP injection of
endometrium Donor Day -7

B

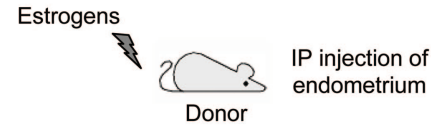

Day -7

C

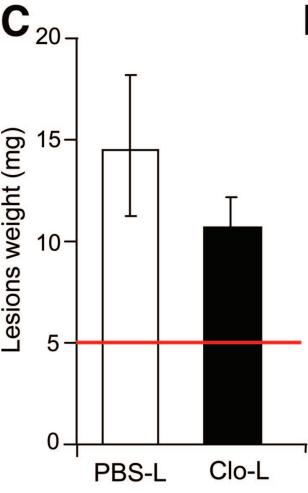

D

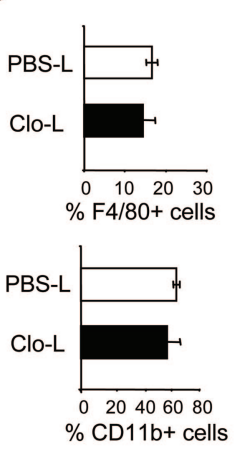

G E\&E CD68 CD31

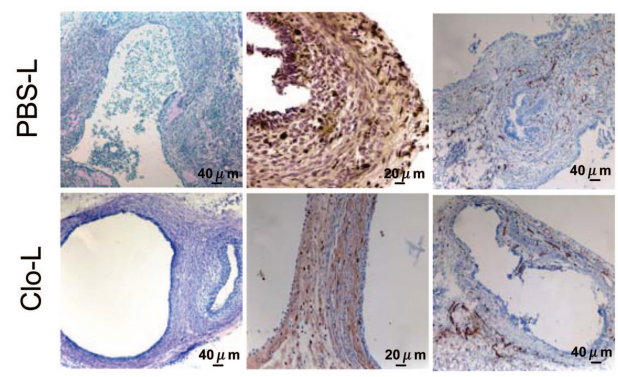

Day 0

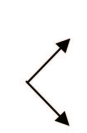

E

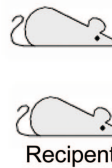

Recipents
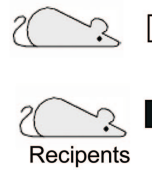

PBS-L
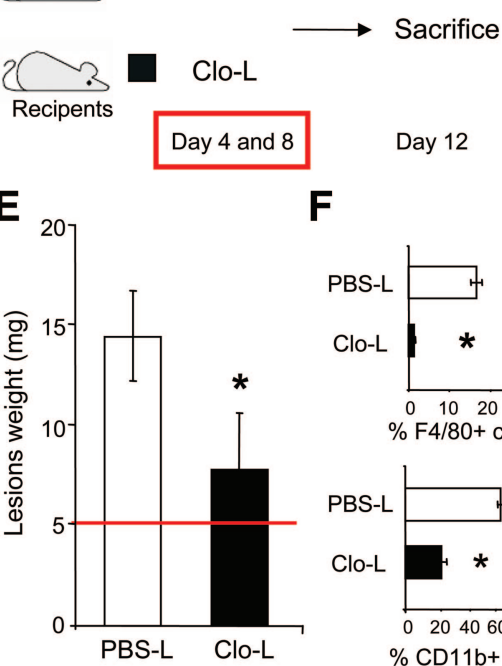

F
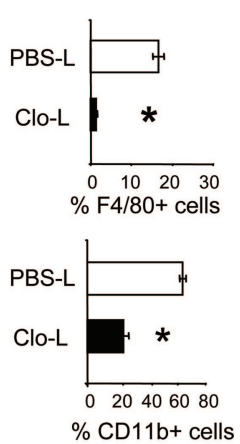

$\mathrm{H}$

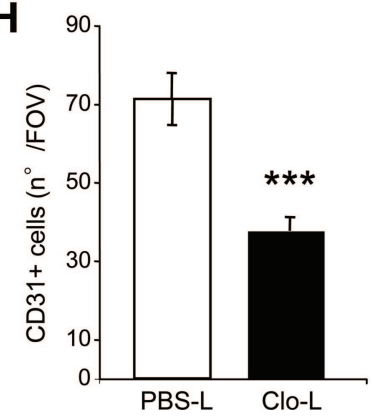

Figure 2. Peritoneal macrophages sustain the growth of established endometriotic lesions. Estradiol benzoate-treated female mice were sacrificed and uteri removed and split. Endometrial tissue was isolated and mechanically disrupted before i.p. injection of an experimental/control mice pair. Control mice (open squares) and depleted mice (filled squares) were treated i.p. with liposomes containing PBS (PBS-L) or clodronate (Clo-L) 24 hours before (A) or 4 and 8 days after (B) i.p. injection of syngeneic endometrial tissue. Lesions were evaluated at day 12 day after endometrium injection. The dry weight of endometriotic lesions from mice treated with PBS-L (open columns) or with Clo-L (filled columns) 24 hours before (C) or 4 and 8 days after (E) i.p. injection of syngeneic endometrial tissue was assessed at day 12. Results are expressed as mean of total weights of lesions from six independent experimental/control mouse pair \pm SEM. F4/ $80^{+}$and $\mathrm{CD} 11 \mathrm{~b}+$ macrophages were identified by flow cytometry in animals treated with PBS-L or Clo-L 24 hours before $(\mathbf{D})$ or 4 and 8 days after $(\mathbf{F})$ i.p. injection of syngeneic endometrial tissue at sacrifice at day 12. Panels report the results expressed as percentage of peritoneal positive cells in mice treated with PBS-L (open columns) or Clo-L (filled columns), G: Macrophages (CD68+ cells) and endothelial cells (CD31+ cells) in the endometriotic lesions obtained from mice treated with PBS-L or with Clo-L were evaluated by immunohistochemistry at day 12 . H: The treatment effect on vascularization was also quantitatively assessed by counting the number of CD31+ cells per field of vision. Results represent the mean \pm SEM of five independent field of vision, as assessed by two independent blinded expert pathologists. Significantly different from control, ${ }^{*} P<$ 0.05 and ${ }^{* * * *} P<0.001$ 


\section{Immunohistochemistry of Human Lesions}

Samples were immediately frozen in optimal cutting temperature compound at $-80^{\circ} \mathrm{C}$. Five- to six-micron thick sections were fixed with $4 \%$ paraformaldehyde for 10 minutes at room temperature, before staining with purified mouse anti-human-CD68, anti-CD163 (Dako), antiCD206 (BD Pharmingen) overnight at room temperature. Biotinylated rat-anti-mouse antibodies were used as second-step reagents. Samples were then processed as described $^{18}$ and examined under a Nikon Eclipse 55i microscope (Nikon, Tokyo, Japan). Images were captured with Digital Sight DS-5 M digital camera (Nikon) using Lucia G software (Laboratory Imaging, Prague, $\mathrm{CZ}$ ). Parallel slides in which primary Ab had been omitted were identically processed and used as negative controls. Infiltration score was calculated independently by two blinded investigators, using grades from 1 to 4 : 1 , no infiltrating macrophages expressing the given marker; 2 , sparse infiltrates, $\leq 5$ infiltrating macrophages expressing the given marker per 0.6-mm lesion core; 3, moderate infiltrates, for $>5$ but $\leq 25$ infiltrating macrophages expressing the given marker per 0.6-mm lesion core, and 4, dense infiltrates, for $>25$ infiltrating macrophages expressing the given marker per 0.6-mm lesion core.

\section{Statistical Analysis}

Statistical analysis was performed using bivariate correlation and linear regression analysis for continuous variable and two-tailed Student's $t$-test for unpaired samples with unequal variance to analyze differences between groups. Analysis was performed with SPSS (11.0 Mac OS $\times$ Version).

\section{Results}

\section{Ectopic Endometrial Lesions Depend on Peritoneal Macrophages for Their Growth and Vascularization}

We compared the establishment of ectopic endometrial lesions in Balb/C mice treated with macrophage-depleting liposomes containing clodronate or with control sham liposomes containing PBS. To minimize the risk of artifacts, mice cohorts were divided in pairs (control and experimental), each receiving identical amounts of endometrial cell suspension from a single syngeneic donor (Figure 1, A). At sacrifice, 12 days after intraperitoneal injection of syngeneic endometrial tissue, lesions had developed in all mice. Depletion was effective, since the fraction of $\mathrm{F} 4 / 80^{+}$and $\mathrm{CD} 11 \mathrm{~b}^{+}$macrophages abated in the peritoneal cavity of mice treated with liposomes containing clodronate, but not in those treated with liposomes containing PBS (Figure 1B). Endometriotic lesions from macrophage-depleted mice were significantly smaller as reflected by their total weight (Figure 1C). To verify that the result of the treatment was due to macrophage depletion, we injected animals 48 hours before intraperitoneal injection of endometrial tissue with a different macrophage-depleting agent, ie, the F4/80 mAb. $\mathrm{mAb}$ injection was then repeated every 48 hours (Figure 1D). Significantly smaller ectopic lesions developed in mice treated with macrophage-depleting mAb than in animals injected with PBS (Figure 1, E-F).

We then assessed whether macrophages were required for the implant of endometrial tissue on the peritoneal membrane and for the ensuing growth of established ectopic lesions. We depleted macrophages with liposomes containing clodronate 24 hours before injection of endometrial tissue (Figure 2A). Peritoneal F4/80 and $\mathrm{CD}_{11} \mathrm{~b}^{+}$macrophages had returned to almost normal levels at the time of sacrifice (day 12), indicating that the effect of the treatment was indeed transient (Figure 2D). Lesions number, weight and microscopic architecture were not apparently affected in depleted animals (Figure 2C).

We also injected syngeneic endometrium at day 0 and treated mice with liposomes containing clodronate or PBS at days 4 and 8 (Figure 2B). Only small numbers of $\mathrm{F} 4 / 80^{+}$and $\mathrm{CD} 11 \mathrm{~b}^{+}$macrophages were detectable in the peritoneal fluid at day 12, when animals were sacrificed, indicating that the depletion was maintained (Figure $2 \mathrm{~F}$ ). The number of the implanted endometriotic lesions did not differ in treated animals (4.83 $\pm 0.3 /$ mouse and $3.33 \pm 0.71 /$ mouse in control and macrophage-de-
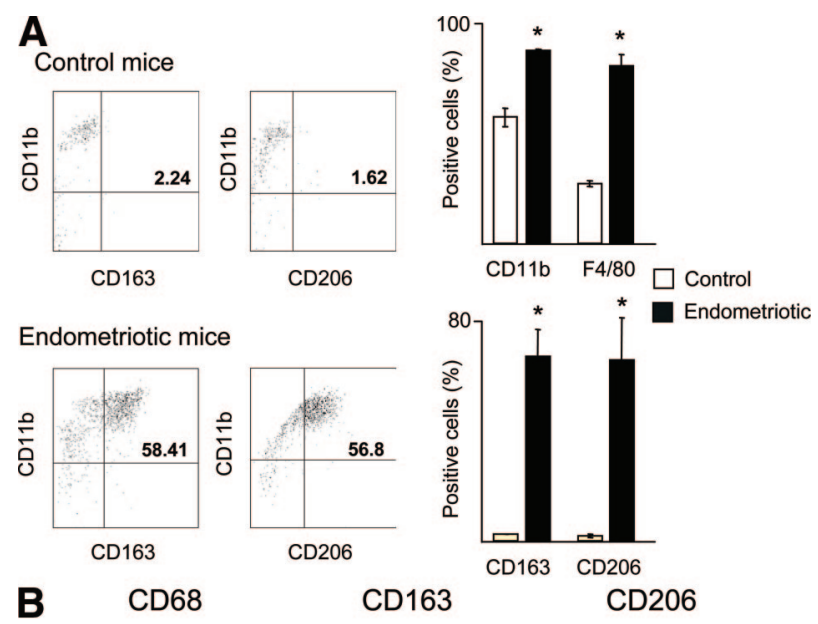

B

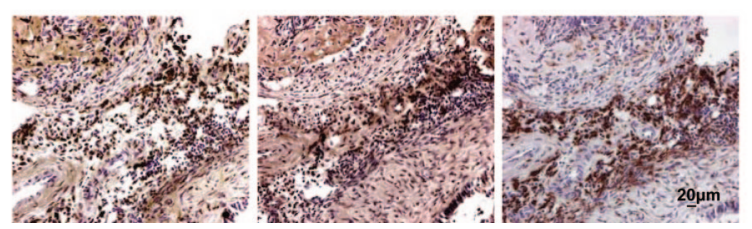

Figure 3. Peritoneal macrophages of mice with established endometriotic lesion express markers of alternative activation. A: Peritoneal cells were retrieved at day 12 from mice injected either with PBS (control mice, open symbols) of from mice 12 days after injection of syngeneic endometrial tissue (endometriotic mice, filled symbols), as described in Materials and Methods Left plots: CD11b + cells ( $y$ axis, red fluorescence) were analyzed by flow cytometry for the expression of markers of alternative activation, such as the CD163 and the CD206 antigens ( $x$ axis, green fluorescence). Right panels: histograms represent the fraction of peritoneal cells expressing each marker in control (open columns) or endometriotic (filled columns) mice. Results represent the mean \pm SEM of three independent experiments. B: Endometrial lesions were characterized by immunohistochemistry for the expression of CD68, CD163, and CD206 in infiltrating macrophages. 
A

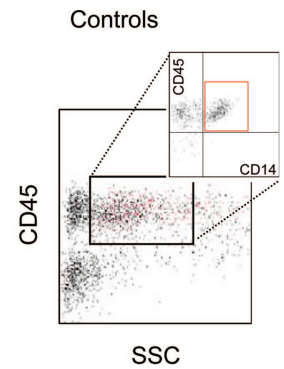

B
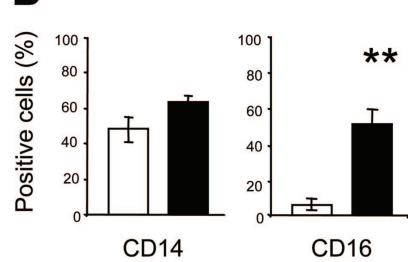

C

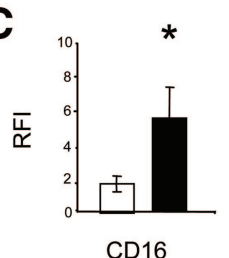

CD16

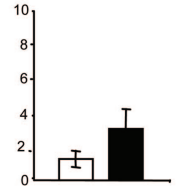

CD163
Endometriotic Patients
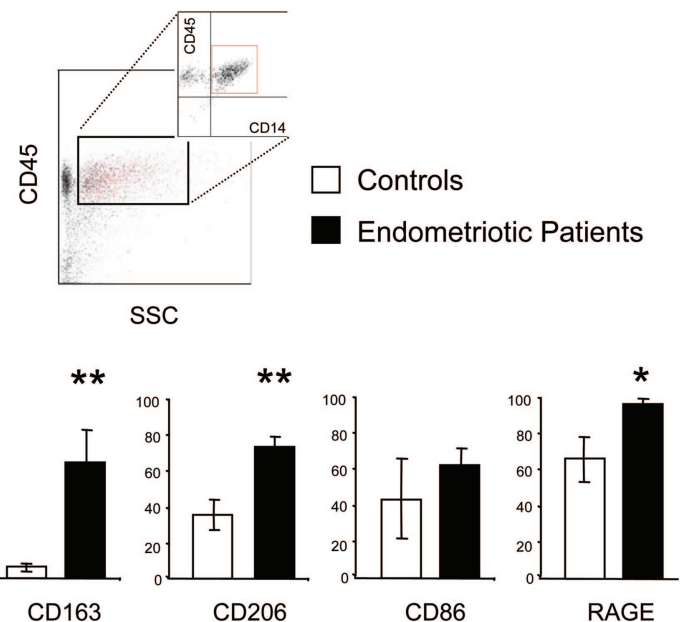

CD86

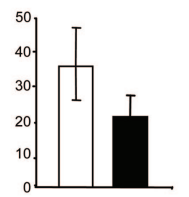

RAGE
Figure 4. Peritoneal macrophages of endometriotic patients express markers of alternative activation. A: Peritoneal cells from endometriotic patients (filled columns) and relevant controls (open columns) were analyzed for the expression of macrophage lineage, activation, and differentiation markers by flow cytometry. The cluster analyzed was defined based on the expression of the CD45 common leukocyte antigen ( $y$ axis, red fluorescence) by cells with appropriate side scatter characteristics ( $x$ axis, green fluorescence) as illustrated. B: $\%$ of peritoneal macrophages expressing the indicated markers in endometriotic patients (filled columns) and relevant controls (open columns). Results are expressed as the mean \pm SEM of 23 endometriotic patients and 13 matched controls C: Expression of indicated markers by peritoneal macrophages from endometriotic patients (filled columns) and relevant controls (open columns) as assessed by the associated relative fluorescence intensity ( $y$ axis) calculated as described in Materials and Methods). Results are expressed as the mean \pm SEM of 23 endometriotic patients and 13 matched controls. Significantly different from control, ${ }^{*} P<0.05$ and ${ }^{* *} P<0.01$ pleted animals respectively), but the total weight of the lesions was significantly lower (Figure 2E). At the microscopic levels, lesions in control mice consisted of dilated glands with columnar or cuboid epithelial endometrial cells, surrounded by narrow stroma layers, with newly formed vessels characterized by $\mathrm{CD} 31^{+}$endothelial cells (Figure 2G). $\mathrm{CD}^{+} 8^{+}$macrophages infiltrated endometriotic lesions (Figure 2G). In contrast, in macrophagedepleted animals, the glandular and stromal architecture was disrupted (Figure 2G), with severely reduced vascularization, as assessed by staining of endothelia with the anti-CD31 mAb (Figure 2, G and H).

\section{Mouse Macrophages in the Peritoneal Fluid and Infiltrating Endometriotic Lesions Express \\ Markers of Alternative Activation}

We derived and analyzed by flow cytometry peritoneal macrophages from control and endometriotic mice. The fraction of peritoneal CD11b + macrophages did not differ in the two groups of mice. In mice with endometriosis, we observed a substantial and significant increase in the fraction of peritoneal macrophages that expressed the F4/80 antigen $\left(96.35 \pm 0.6 \%\right.$ of $C D 11 b^{+}$cells; $P=0.002$ vs controls). CD11 $\mathrm{b}^{+}$macrophages from the peritoneal liquid of endometriotic mice co-expressed markers of alternative activation, the hemoglobin scavenger receptor, CD163 and the mannose receptor, CD206, which were virtually absent in CD11 $\mathrm{b}^{+}$macrophages of control mice $\left(58.41 \pm 8.4\right.$ of $\mathrm{CD}_{163}{ }^{+}, 56.8 \pm 13.8$ of $\mathrm{CD}^{2} 26^{+}$ cells; $P=0.01$ and 0.03 respectively versus controls) (Figure 3, A and B). CD68 ${ }^{+}$macrophages infiltrating endometriotic lesions expressed the CD163 and the CD206 receptors (Figure 3B), while those associated to the peritoneal tissue of control mice were next to negative (not shown).

\section{Macrophages in the Peritoneal Fluid of Endometriotic Patients or Infiltrating Lesions} Express Markers of Alternative Activation

To verify the relevance of the results obtained in the mouse model for the human disease, we analyzed peritoneal cells and tissues from 21 consecutive endometriotic patients and 12 matched controls. To unambiguously identify macrophages in the peritoneal fluid, flow cytometric analysis has been performed on the cluster depicted in Figure 4A, in which events with congruent side scatter physical characteristics were selected among cells expressing the CD45 common leukocyte antigen. Cells were then analyzed for the expression of lineage and activation markers, as depicted in Figure 4A for the CD14 receptor. While CD14+ peritoneal macrophages were similar in patients and controls, $\mathrm{CD}^{+} 6^{+}$cells were $50.04 \pm 8.6$ of macrophages in endometriotic patients and $5.68 \pm 3.2$ in control subjects $(P<0.01)$; macrophages expressing the CD163 hemoglobin scavenger receptor were $19.04 \pm 5.4$ in patients and $1.75 \pm 0.56$ in controls $(P<0.01)$; macrophages expressing the CD206 mannose receptor were $68.82 \pm 5.6$ in patients and $33.68 \pm 7.6$ in controls $(P<0.01)$ (Figure 4B). The fraction of peritoneal macrophages from endometriotic patients and control subjects that express the CD86 receptor (Figure 4B) or the MHC class I and II, $\alpha \vee \beta 3, \mathrm{CD} 40$, and CD36 molecules was similar (not shown), while the difference in the fraction of anti-receptor for advanced glycation end products-expressing macrophages, although significant, was modest (Figure 4B). The level of 
expression of each molecule, as assessed by the associated relative fluorescence intensity, was similar in CD14+ peritoneal macrophages from patients and controls, with the exception of CD16, whose expression was significantly higher in patients (relative fluorescence intensity was $6.89 \pm 2.3$ in patients and $2.37 \pm 0.66$ in controls, $P<0.05$; Figure $4 C$ ). The differences were specifically associated to the peritoneal environment, because we failed to detect any significant difference between the expression of these markers in circulating leukocytes from patients and controls (not shown). We did not observe any correlation between the expression of markers of macrophage alternative activation and the clinical stage of the disease. ${ }^{19}$ However, only five patients with mild endometriosis (stages I-II) were studied. Data on a larger cohort of patients will be necessary to draw conclusions on the influence of endometriosis stage on this parameter.

The immunohistochemical study confirmed that $\mathrm{CD}^{+} 8^{+}$macrophages preferentially infiltrate endometriotic lesions, even if they can be detected also in the disease-free peritoneum of patients and controls and in uterine leiomyomas. Endometriotic macrophages only expressed the CD163 and CD206 receptors, which were virtually absent in control tissues (Figure $5 \mathrm{~A}$ ). The difference was statistically significant $(P<0.05$; Figure $5 B)$.

\section{Opposite Effect of M1 and M2 Macrophages on Endometriotic Lesions Growth}

To directly address the role of macrophage activation and polarization in vivo we propagated from syngeneic bone marrow precursors either undifferentiated MO macrophages or cells polarized toward an M1 inflammatory or M2 alternatively activated macrophages (Figure 6A). M0 cells did not secrete significant amounts of cytokines. In contrast M1 macrophages produced significant amounts of TNF- $\alpha$ and negligible amount of IL-10. The opposite was true for M2 macrophages, which secreted IL-10 but not TNF- $\alpha$ (Figure 6B). M0, M1, and M2 macrophages were transferred in the peritoneum of recipient mice 2 days before and at the time of the injection of endometrium. Macrophage transfer was repeated 2 days after. Non differentiated M0 macrophages did not influence the establishment and the growth of endometrial lesions, as assessed at the time of animal sacrifice. In contrast lesions growth was significantly reduced in the presence of M1 macrophages (Figure 6C) and significantly enhanced in the presence of M2 macrophages (Figure 6C). Lesions in mice injected with $\mathrm{M} 1$ macrophages were barely detectable and exhibited a severely disrupted architecture (Figure 6D).

\section{Discussion}

Macrophages play a critical role, via their ability to guide tissue regeneration, in the growth of tumors and in diseases characterized by persistent tissue remodeling. ${ }^{9,20-25}$ Several lines of evidence suggest that macro-
A
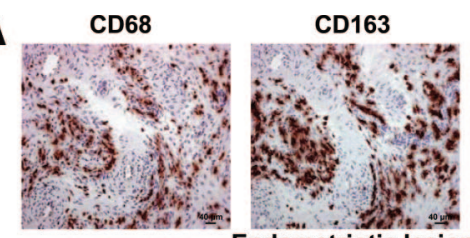
CD206

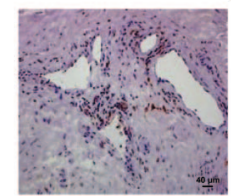

Endometriotic lesion
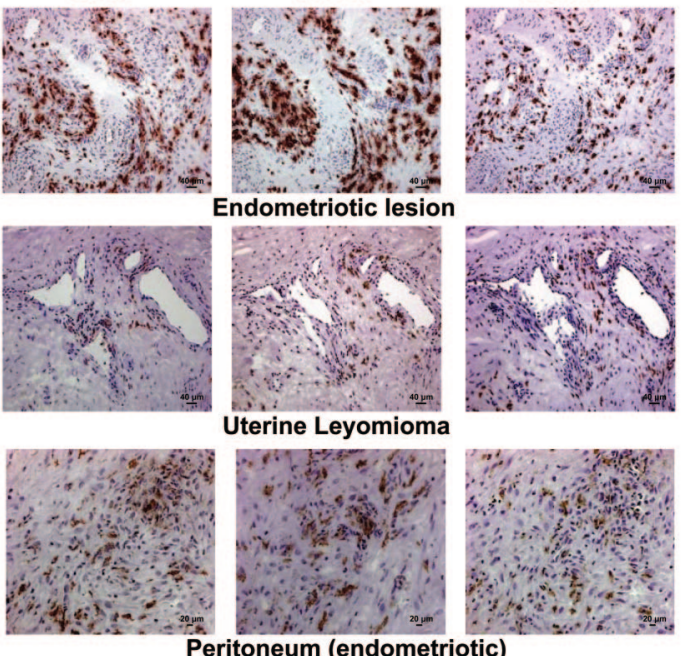

Uterine Leyomioma
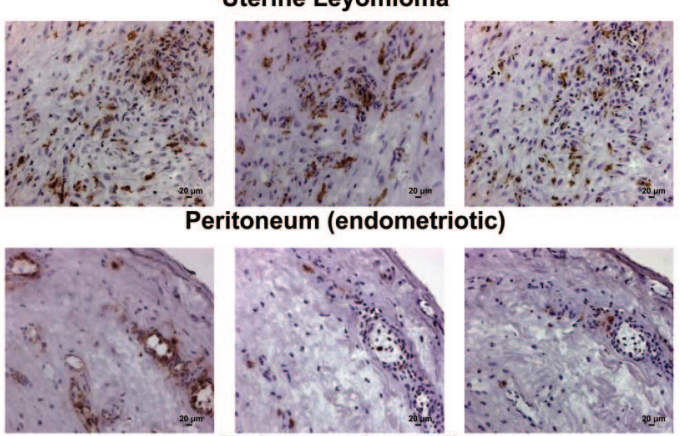

eritoneum (endometriotic)
B
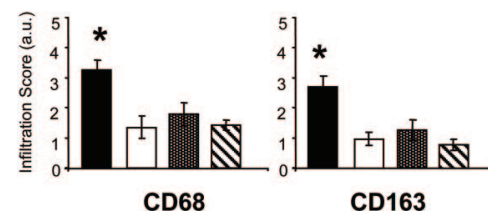

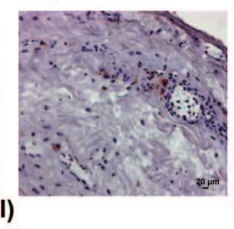

Peritoneum (control)

Endometriotic lesion $\square$ Uterine leyomioma 圈 Peritoneum (endometriotic) $\mathbf{Q}$ Peritoneum (control)
Figure 5. Macrophages infiltrating endometriotic lesions and apparently healthy peritoneum express markers of alternative activation. A: Macrophages in endometriotic lesions, leiomyomas, and apparently nonaffected peritoneum from patients with endometriosis and controls were characterized by immunohistochemistry for the expression of a lineage marker (CD68) and for markers of alternative activation (CD163 and CD206). B: Infiltrating macrophages expressing CD68, CD163, and CD206 were quantitatively assessed by the infiltration score, performed as described in Materials and Methods. ${ }^{*} P<0.05$, significantly different from control.

phages are locally activated and not only "trapped" in ectopic endometrial lesions. ${ }^{1,26-31}$ For example, the nuclear factor $-\kappa \mathrm{B}$ transcription factor is activated in macrophages from endometriotic patients ${ }^{27}$ and responsive genes that determine macrophages functions undergo transactivation, supporting angiogenesis and tissue remodeling ${ }^{32,33}$ Accordingly, the in vivo inhibition of nuclear factor- $\kappa \mathrm{B}$ interferes with the growth of experimental lesions. ${ }^{34}$ The environmental cues that activate peritoneal macrophages are still uncertain: local hypoxia apparently plays a major role. ${ }^{35,36}$ Aging erythrocytes, accumulating and dying into the pelvic cavity because of retrograde menstruation, cause iron overload. ${ }^{37}$ Iron overload is greater in peritoneal macrophages, which are in charge of erythrocytes phagocytic clearance. ${ }^{20,38}$ Iron overload could as well influence macrophage activation.

Our goal was to verify whether macrophage activation is an epiphenomenon or is causally related to endometriosis. We deleted cells before and at different times after intraperitoneal injection of syngeneic endometrium in ex- 
A

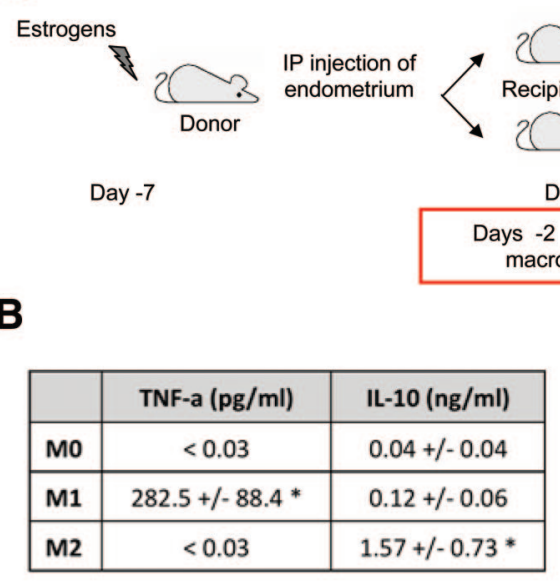

B

\begin{tabular}{|c|c|c|}
\hline & TNF-a (pg/ml) & IL-10 (ng/ml) \\
\hline M0 & $<0.03$ & $0.04+/-0.04$ \\
\hline M1 & $282.5+/-88.4^{*}$ & $0.12+/-0.06$ \\
\hline M2 & $<0.03$ & $1.57+/-0.73^{*}$ \\
\hline
\end{tabular}

D
D $\quad E \& E$

M1

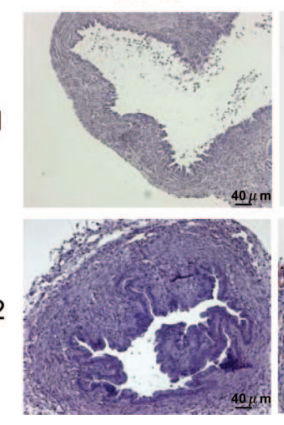

CD68

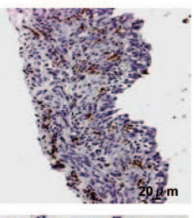

Day 0

$\begin{array}{lll}-2 & 0 & +2\end{array}$

\section{C}

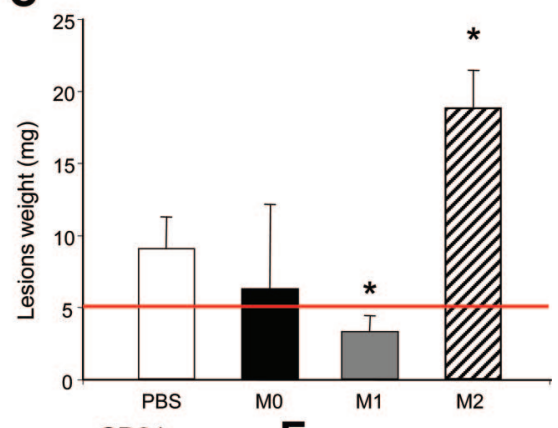

E

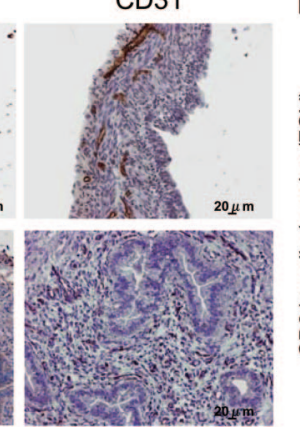

Day 12

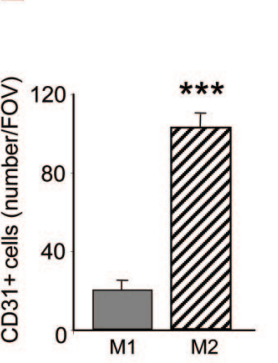

Figure 6. Endometriotic lesions growth is dependent on macrophages activation. A: Estradiol benzoate-treated female mice were sacrificed and uteri were removed and split. Endometrial tissue was isolated and mechanically disrupted before i.p. injection of four experimental/control mice pair per each treatment group. Control mice were treated with PBS. Experimental animals were injected i.p. at day -2 , 0 , and +2 with $\mathrm{M} 0, \mathrm{M} 1$, and M2 macrophages $\left(1.5 \times 10^{6} \mathrm{cells} /\right.$ mouse $\left./ 100 \mu \mathrm{l}\right)$. Animals were sacrificed 12 days after endometrial tissue injection. B: TNF- $\alpha$ and IL-10 secretion by in vitro polarized M0, M1, and M2 macrophages was assessed by enzyme-linked immunosorbent assay. Results are expressed as the mean \pm SEM of at least three independent experiments. C: The dry weight of endometriotic lesions was assessed in mice treated with PBS or injected with M0, M1, or M2 macrophages. Results are expressed as mean of total weights of lesions from at leas four mice \pm SEM. ${ }^{*} P<0.05$, significantly different from control group. D: Representative H\&E staining and immunohistochemical analysis for $\mathrm{CD} 68+$ cells in endometriotic lesions obtained from mice treated with M1 and M2 macrophages. perimental animals. Results in Figures 1-2 indicate that endogenous peritoneal macrophages are required for the experimental model of the human disease. Accordingly, a recent study has shown that the depletion of macrophages in the rat peritoneum by local injection of liposomal alendronate reduces both the initiation and the growth of endometriotic lesions. ${ }^{39}$

The establishment of endometriotic lesions is characterized by infiltration of the underlying tissue and attraction of novel vessels, with development of an organized ectopic glandular and of stromal architecture. The latter events are disrupted in the absence of macrophages (Figures 1-2). Macrophages deliver trophic and antiapoptotic signals, in particular during the very first days after endometrium injection, ${ }^{40}$ before the formation of novel vessels, ${ }^{36,41,42}$ which possibly facilitate the survival of ectopic cells in a relatively hypoxic environment. ${ }^{40}$

Endometriotic macrophages are actively involved in both i) the phagocytic clearance of aged red cells 20,38 and endometrial cell debris, ${ }^{43}$ and ii) the secretion of trophic and neo-angiogenic molecules. These features are typical of macrophages that have acquired an alternatively activated phenotype. ${ }^{8,44}$ We addressed this possibility characterizing membrane receptors expressed by macrophages in the peritoneal fluid and in the tissues of endometriosis patients and of experimental mice. We focused on two markers of M2-polarized macrophages, the CD206 man- nose receptor and the CD163 hemoglobin scavenger receptor. ${ }^{45}$ CD206 belongs to the C-type lectin superfamily, behaves as a pattern recognition receptor and has been involved in the silent clearance of inflammatory signals ${ }^{46}$ CD163 physiologically scavenges haptoglobin by inducing endocytosis of haptoglobin-hemoglobin complexes, with degradation and consequent rescue of heme-iron components for erythropoiesis. ${ }^{47}$ Its up-regulation reveals vascular defect and haptoglobin-hemoglobin complex stimulation in vivo. ${ }^{48}$

Peritoneal macrophages express high levels of both markers both in patients and mice (Figures 3-4). Both macrophages in the peritoneal fluid and macrophages actively infiltrating ectopic lesions display features of alternative activation (Figures $3-4$ ). This suggests that signals in the peritoneal cavity of endometriotic subjects and restricted to the ectopic tissue, switch the differentiation program of endogenous blood-derived precursors toward an alternative reparative phenotype. In turn, alternatively activated macrophages are responsible for lesions vascularization and growth.

We directly addressed this model by adoptive transfer of inflammatory/M1 and alternatively activated/M2 macrophages. The treatment resulted in a dramatically impaired or enhanced establishment of endometriotic lesions respectively (Figure 6). This effect probably depends on several features of polarized macrophages including 
their ability to secrete different arrays of soluble factors (Figure 6). In support to this model, the peritoneal fluid of patients with endometriosis interferes with in vitro differentiation of precursors challenged with recombinant cytokines, favoring their differentiation toward macrophages $^{9}$ and immature dendritic cells injected into endometriotic mice intraperitoneally actively migrate and infiltrate ectopic endometrial lesions, where they contribute to lesions further development. ${ }^{49}$

In conclusion, our findings indicate that the peritoneal environment controls the differentiation of macrophage precursors, committing them toward an alternatively activated, reparatory phenotype. In turn, alternatively activated macrophages are necessary for ectopic lesions to vascularize and grow. These results are important for several reasons. First, they suggest that the alternative activation of macrophages is a clue to the heterogeneity in the susceptibility to endometriosis among menstruating women, especially in light of human data showing how environmental stimuli have shaped the genetic variations of our innate response, possibly originating complex individual responses to infectious agents ${ }^{11}$ but also endogenous alarm signals. Second, they suggest that it may be possible to treat human endometriosis though local inhibition of macrophage reparative actions.

\section{References}

1. Lebovic DI, Mueller MD, Taylor RN: Immunobiology of endometriosis Fertil Steril 2001, 75:1-10

2. Giudice LC, Kao LC: Endometriosis. Lancet 2004, 364:1789-1799

3. Berkley KJ, Rapkin AJ, Papka RE: The pains of endometriosis. Science 2005, 308:1587-1589

4. Sampson J: Peritoneal endometriosis due to menstrual dissemination of endometrial tissue into the peritoneal cavity. Am J Obstet Gynecol 1927, 14:422-469

5. Dunselman GA, Groothuis PG: Etiology of endometriosis: hypotheses and facts. Gynecol Obstet Invest 2004, 57:42-43

6. Becker CM, D'Amato RJ: Angiogenesis and antiangiogenic therapy in endometriosis. Microvasc Res 2007, 74:121-130

7. van Amerongen MJ, Harmsen MC, van Rooijen N, Petersen AH, van Luyn MJ: Macrophage depletion impairs wound healing and increases left ventricular remodeling after myocardial injury in mice. Am J Pathol 2007, 170:818-829

8. Martinez FO, Sica A, Mantovani A, Locati M: Macrophage activation and polarization. Front Biosci 2008, 13:453-461

9. Allavena P, Sica A, Solinas G, Porta C, Mantovani A: The inflammatory micro-environment in tumor progression: the role of tumor-associated macrophages. Crit Rev Oncol Hematol 2008, 66:1-9

10. Ferwerda B, McCall MB, Alonso S, Giamarellos-Bourboulis EJ, Mouktaroudi M, Izagirre N, Syafruddin D, Kibiki G, Cristea T, Hijmans A, Hamann L, Israel S, ElGhazali G, Troye-Blomberg M, Kumpf O, Maiga B, Dolo A, Doumbo O, Hermsen CC, Stalenhoef AF, van Crevel R, Brunner HG, Oh DY, Schumann RR, de la Rua C, Sauerwein R, Kullberg BJ, van der Ven AJ, van der Meer JW, Netea MG: TLR4 polymorphisms, infectious diseases, and evolutionary pressure during migration of modern humans. Proc Natl Acad Sci USA 2007, 104:16645-16650

11. Ferwerda B, McCall MB, Verheijen K, Kullberg BJ, van der Ven AJ, Van der Meer JW, Netea MG: Functional consequences of toll-like receptor 4 polymorphisms. Mol Med 2008, 14:346-352

12. Wu MH, Shoji Y, Wu MC, Chuang PC, Lin CC, Huang MF, Tsai SJ: Suppression of matrix metalloproteinase- 9 by prostaglandin $E(2)$ in peritoneal macrophage is associated with severity of endometriosis. Am J Pathol 2005, 167:1061-1069

13. Somigliana E, Vigano P, Rossi G, Carinelli S, Vignali M, PaninaBordignon P: Endometrial ability to implant in ectopic sites can be prevented by interleukin- 12 in a murine model of endometriosis. Hum Reprod 1999, 14:2944-2950

14. Somigliana E, Vigano P, Filardo P, Candiani M, Vignali M, PaninaBordignon P: Use of knockout transgenic mice in the study of endometriosis: insights from mice lacking beta(2)-microglobulin and interleukin-12p40. Fertil Steril 2001, 75:203-206

15. Brignole C, Pastorino F, Marimpietri D, Pagnan G, Pistorio A, Allen TM, Pistoia V, Ponzoni M: Immune cell-mediated antitumor activities of GD2-targeted liposomal c-myb antisense oligonucleotides containing CpG motifs. J Natl Cancer Inst 2004, 96:1171-1180

16. Lolmede K, Campana L, Vezzoli M, Bosurgi L, Tonlorenzi R, Clementi E, Bianchi ME, Cossu G, Manfredi AA, Brunelli S, Rovere-Querini P: Inflammatory and alternatively activated human macrophages attract vessel-associated stem cells, relying on separate HMGB1- and MMP9-dependent pathways. J Leukoc Biol 2009, 85:779-787

17. Revised American Fertility Society classification of endometriosis: 1985. Fertil Steril 1985, 43:351-352

18. Akoum A, Jolicoeur C, Kharfi A, Aube M: Decreased expression of the decoy interleukin-1 receptor type II in human endometriosis. Am J Pathol 2001, 158:481-489

19. Revised American Society for Reproductive Medicine classification of endometriosis: 1996. Fertil Steril 1997, 67:817-821

20. Brunelli S, Rovere-Querini P: The immune system and the repair of skeletal muscle. Pharmacol Res 2008, 58:117-121

21. MacKinnon AC, Farnworth SL, Hodkinson PS, Henderson NC, Atkinson KM, Leffler H, Nilsson UJ, Haslett C, Forbes SJ, Sethi T: Regulation of alternative macrophage activation by galectin-3. J Immunol 2008, 180:2650-2658

22. Arnold L, Henry A, Poron F, Baba-Am Y, van Rooijen N, Plonquet A, Gherardi RK, Chazaud B: Inflammatory monocytes recruited after skeletal muscle injury switch into antiinflammatory macrophages to support myogenesis. J Exp Med 2007, 204:1057-1069

23. Kamada N, Hisamatsu T, Okamoto S, Chinen H, Kobayashi T, Sato T, Sakuraba A, Kitazume MT, Sugita A, Koganei K, Akagawa KS, Hibi T: Unique CD14 intestinal macrophages contribute to the pathogenesis of Crohn disease via IL-23/IFN-gamma axis. J Clin Invest 2008, 118:2269-2280

24. Kelly J, Ali Khan A, Yin J, Ferguson TA, Apte RS: Senescence regulates macrophage activation and angiogenic fate at sites of tissue injury in mice. J Clin Invest 2007, 117:3421-3426

25. Lumeng CN, Bodzin JL, Saltiel AR: Obesity induces a phenotypic switch in adipose tissue macrophage polarization. J Clin Invest 2007, 117:175-184

26. Lawson C, Al-Akoum M, Maheux R, Akoum A: Increased expression of interleukin-1 receptor type 1 in active endometriotic lesions. Reproduction 2007, 133:265-274

27. Lousse JC, Van Langendonckt A, Gonzalez-Ramos R, Defrere S, Renkin E, Donnez J: Increased activation of nuclear factor-kappa B (NF-kappaB) in isolated peritoneal macrophages of patients with endometriosis. Fertil Steril 2008, 90:217-220

28. Minici F, Tiberi F, Tropea A, Fiorella M, Orlando M, Gangale MF, Romani F, Catino S, Campo S, Lanzone A, Apa R: Paracrine regulation of endometriotic tissue. Gynecol Endocrinol 2007, 23:574-580

29. Zhang C, Maeda N, Izumiya C, Yamamoto Y, Kusume T, Oguri H, Yamashita C, Nishimori Y, Hayashi K, Luo J, Fukaya T: Killer immunoglobulin-like receptor and human leukocyte antigen expression as immunodiagnostic parameters for pelvic endometriosis. Am J Reprod Immunol 2006, 55:106-114

30. Galleri L, Luisi S, Rotondi M, Romagnani P, Cobellis L, Serio M, Petraglia F: Low serum and peritoneal fluid concentration of interferon-gamma-induced protein-10 (CXCL10) in women with endometriosis. Fertil Steril 2008, 91:331-334

31. Herrmann Lavoie C, Fraser D, Therriault MJ, Akoum A: Interleukin-1 stimulates macrophage migration inhibitory factor secretion in ectopic endometrial cells of women with endometriosis. Am J Reprod Immunol 2007, 58:505-513

32. Timmer AM, Nizet V: IKKbeta/NF-kappaB and the miscreant macrophage. J Exp Med 2008, 205:1255-1259

33. Hagemann T, Lawrence T, McNeish I, Charles KA, Kulbe H, Thompson RG, Robinson SC, Balkwill FR: "Re-educating" tumor-associated macrophages by targeting NF-kappaB. J Exp Med 2008, 205:1261-1268

34. Gonzalez-Ramos R, Van Langendonckt A, Defrere S, Lousse JC, Mettlen M, Guillet A, Donnez J: Agents blocking the nuclear factor- 
kappaB pathway are effective inhibitors of endometriosis in an in vivo experimental model. Gynecol Obstet Invest 2008, 65:174-186

35. Wu MH, Chen KF, Lin SC, Lgu CW, Tsai SJ: Aberrant expression of leptin in human endometriotic stromal cells is induced by elevated levels of hypoxia inducible factor-1alpha. Am J Pathol 2007, 170:590-598

36. Becker CM, Rohwer N, Funakoshi T, Cramer T, Bernhardt W, Birsner A, Folkman J, D'Amato RJ: 2-methoxyestradiol inhibits hypoxia-inducible factor- 1 \{alpha\} and suppresses growth of lesions in a mouse model of endometriosis. Am J Pathol 2008, 172:534-544

37. Defrere S, Lousse JC, Gonzalez-Ramos R, Colette S, Donnez J, Van Langendonckt A: Potential involvement of iron in the pathogenesis of peritoneal endometriosis. Mol Hum Reprod 2008, 14:377-385

38. Lousse JC, Defrere S, Van Langendonckt A, Gras J, Gonzalez-Ramos $\mathrm{R}$, Colette S, Donnez J: Iron storage is significantly increased in peritoneal macrophages of endometriosis patients and correlates with iron overload in peritoneal fluid. Fertil Steril 2008, 91:16681675

39. Haber E, Danenberg HD, Koroukhov N, Ron-El R, Golomb G, Schachter M: Peritoneal macrophage depletion by liposomal bisphosphonate attenuates endometriosis in the rat model. Hum Reprod 2009, 24:398-407

40. Lin YJ, Lai MD, Lei HY, Wing LY: Neutrophils and macrophages promote angiogenesis in the early stage of endometriosis in a mouse model. Endocrinology 2006, 147:1278-1286

41. Grummer R: Animal models in endometriosis research. Hum Reprod Update 2006, 12:641-649

42. Eggermont J, Donnez J, Casanas-Roux F, Scholtes H, Van Langendonckt
A: Time course of pelvic endometriotic lesion revascularization in a nude mouse model. Fertil Steril 2005, 84:492-499

43. Harada T, Taniguchi F, Izawa M, Ohama Y, Takenaka Y, Tagashira Y Ikeda A, Watanabe A, Iwabe T, Terakawa N: Apoptosis and endometriosis. Front Biosci 2007, 12:3140-3151

44. Gordon S: The macrophage: past, present and future. Eur J Immunol 2007, 37 Suppl 1:S9-S17

45. Verreck FA, de Boer T, Langenberg DM, van der Zanden L, Ottenhoff $\mathrm{TH}$ : Phenotypic and functional profiling of human proinflammatory type-1 and anti-inflammatory type-2 macrophages in response to microbial antigens and IFN-gamma- and CD40L-mediated costimulation. J Leukoc Biol 2006, 79:285-293

46. Allavena P, Chieppa M, Monti P, Piemonti L: From pattern recognition receptor to regulator of homeostasis: the double-faced macrophage mannose receptor. Crit Rev Immunol 2004, 24:179-192

47. Kristiansen M, Graversen JH, Jacobsen C, Sonne O, Hoffman HJ Law SK, Moestrup SK: Identification of the haemoglobin scavenger receptor. Nature 2001, 409:198-201

48. Borda JT, Alvarez X, Mohan M, Hasegawa A, Bernardino A, Jean S, Aye P, Lackner AA: CD163, a marker of perivascular macrophages, is up-regulated by microglia in simian immunodeficiency virus encephalitis after haptoglobin-hemoglobin complex stimulation and is suggestive of breakdown of the blood-brain barrier. Am J Pathol 2008, 172:725-737

49. Fainaru O, Adini A, Benny O, Adini I, Short S, Bazinet L, Nakai K Pravda E, Hornstein MD, D'Amato RJ, Folkman J: Dendritic cells support angiogenesis and promote lesion growth in a murine model of endometriosis. FASEB J 2008, 22:522-529 